\title{
DYNAMICAL TRANSITION THEORY OF HEXAGONAL PATTERN FORMATIONS
}

\author{
TAYLAN ŞENGÜL
}

\begin{abstract}
The main goal of this paper is to understand the formation of hexagonal patterns from the dynamical transition theory point of view. We consider the transitions from a steady state of an abstract nonlinear dissipative system. To shed light on the formation of mixed mode patterns such as the hexagonal pattern, we consider the case where the linearized operator of the system has two critical real eigenvalues, at a critical value $\lambda_{c}$ of a control parameter $\lambda$ with associated eigenmodes having a roll and rectangular pattern. By using center manifold reduction, we obtain the reduced equations of the system near the critical transition value $\lambda_{c}$. By a through analysis of these equations, we fully characterize all possible transition scenarios when the coefficients of the quadratic part of the reduced equations do not vanish. We consider three problems, two variants of the 2D Swift-Hohenberg equation and the 3D surface tension driven convection, to demonstrate that all the main theoretical results we obtain here are indeed realizable.
\end{abstract}

\section{Introduction: Main assumptions And RESUlts}

Transition phenomena is throughout all nonlinear sciences [23, 16, 14]. It shapes many physical, biological and social systems through instabilities. The formation of patterns in such systems, whether it be coatings of animals [24], convection cells in fluid systems [1] or crime patterns in cities [35], is intrinsically related to the transitions taking place in those systems. One of the tools to understand and classify the transition behavior is the dynamic transition theory [23]. The current work is an attempt to combine this theory with certain aspects of pattern formations and relies on some of the previous work in this direction [10, 33, 34, 20].

1.1. The setting and the main assumptions. We are interested in the transitions of a steady state solution of a general nonlinear dissipative system [37] on a Hilbert space $X$

$$
\frac{d u}{d t}=L_{\lambda} u+G_{\lambda}(u), \quad t>0
$$

where $u:[0, \infty) \mapsto X$ is the unknown function and $\lambda \in \mathbb{R}^{1}$ is a parameter. Here $L_{\lambda}: X_{1} \rightarrow X$ is a linear operator where $X_{1}$ is another 
Banach space with compact and dense inclusion $X_{1} \subset X$ and $G_{\lambda}$ is a nonlinear operator satisfying certain properties given later.

1.1.1. The assumptions on the spectrum of the linear operator. We will assume that the linear operator $L_{\lambda}$ has a countably infinite set of eigenvalues

$$
\left\{\beta_{i}(\lambda) \in \mathbb{C}: i \in \mathbb{N}\right\}
$$

with a complete set of eigenvectors

$$
\left\{f_{i} \in X_{1}, i=1,2, \ldots\right\}
$$

satisfying the following conditions on its spectrum, known as the PES conditions.

$$
\begin{aligned}
& \beta_{1}(\lambda), \beta_{2}(\lambda) \in \mathbb{R}, \\
& \beta(\lambda):=\beta_{1}(\lambda)=\beta_{2}(\lambda) \begin{cases}<0 & \lambda<\lambda_{c} \\
=0 & \lambda=\lambda_{c} \\
>0 & \lambda>\lambda_{c}\end{cases} \\
& \operatorname{Re} \beta_{i}<0, \quad \forall i=3,4, \ldots
\end{aligned}
$$

Much of the linear theory on stability and transitions involves establishing the PES conditions, see [2] for the classical fluid dynamics and [28] for the geophysical fluid dynamics.

1.1.2. The assumptions on the physical space. As the physical space, we assume a bounded spatial domain with at least two dimensions. We also distinguish between the eigenvectors $f_{i}$ of the linear operator and a (possibly) distinct set of basis vectors

$$
\left\{e_{i_{1}, i_{2}}^{j} \in X_{1}: i_{1}, i_{2}, j \in \mathbb{N}\right\}
$$

indexed by the wave indices $i_{1}, i_{2}$ spanning the two horizontal spatial dimensions and $j$ is the index of the other directions which we usually suppress for ease of notation. Moreover, we assume that the first two critical modes have the spatial structure

$$
f_{1} \sim e_{j_{1}, j_{2}}, \quad f_{2} \sim e_{k_{1}, 0},
$$

for some non-negative integers $j_{1}, j_{2}, k_{1}$. Even for 2D problems, the basis vectors $e_{i, j}$ are usually different from the basis vectors $f_{j}$ of the linear operator. For example, in a scalar reaction diffusion type equation, the vectors $e_{i, j}$ are usually the eigenbasis of the Laplacian operator with the given boundary conditions, while for fluid problems, the vectors $e_{i, j}$ are the eigenbasis of the Stokes operator, as we consider in Section 3.3.

As an example, if the spatial domain of interest is a rectangular domain $\left(0, L_{1} \pi\right) \times\left(0, L_{2} \pi\right) \times(0,1)$ with Neumann boundary conditions in the horizontal directions, then the eigenmodes $f_{j}$ are given by

$$
f_{j}=\sum_{k=1}^{\infty} \hat{f}_{k} e_{j_{1}, j_{2}}^{j}(x, y, z)
$$


where

$$
e_{j_{1}, j_{2}}^{j}=\cos \left(j_{1} x_{1} / L_{1}\right) \cos \left(j_{2} x_{2} / L_{2}\right) w_{j}(z)
$$

where $j_{1}, i_{2}$ are non-negative integers and $w_{j}$ are basis functions satisfying the vertical boundary conditions.

Under the assumption (3), the eigenmode $f_{1}$ represents a rectangular (horizontal) pattern, and the eigenmode $f_{2}$ represents a roll pattern. Their linear combinations give rise to mixed patterns such as the hexagonal pattern, see Figure 1.
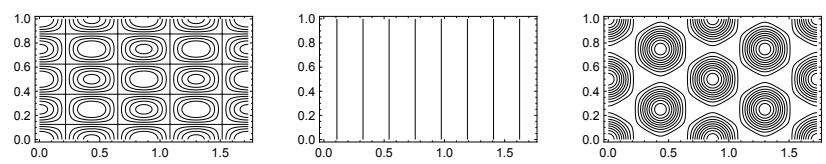

Figure 1. (a) A rectangular mode $\cos \left(\frac{4 x_{1}}{\sqrt{3}}\right) \cos \left(4 x_{2}\right)$

(b) A roll mode $\cos \left(\frac{8 x_{1}}{\sqrt{3}}\right)$ (c) A mixed mode $4 \cos \left(\frac{4 x_{1}}{\sqrt{3}}\right) \cos \left(4 x_{2}\right)+\cos \left(\frac{8 x_{1}}{\sqrt{3}}\right)$.

In many physical examples, the critical modes are selected according to the horizontal wave number, that is the first two critical modes have equal horizontal wave numbers. This implies, (3) means

$$
\left(\frac{j_{1} \pi}{L_{1}}\right)^{2}+\left(\frac{j_{2} \pi}{L_{2}}\right)^{2}=\left(\frac{k_{1} \pi}{L_{1}}\right)^{2}
$$

from which $k_{1}>j_{1}$ follows. We remark here that, the equality of the wave numbers of the first two critical modes, imposes a severe relation on the horizontal aspect ratio $L_{2} / L_{1}$ of a rectangular domain.

$$
L_{1}=\sqrt{\frac{k_{1}^{2}-j_{1}^{2}}{j_{2}^{2}}} L_{2} .
$$

As a result, in applications, this type of transition is non-generic, that is, does not occur if the aspect ratio is chosen randomly. We give the choice of the wave indices for the 3D Rayleigh-Benard convection with free slip boundary conditions in Figure 2. The figure shows the nongenericity of the higher multiplicity transitions and the length scales at which double equal wave number mode transitions occur for (a) a roll and a rectangle mode, (b) two roll modes, (c) two rectangle modes. 


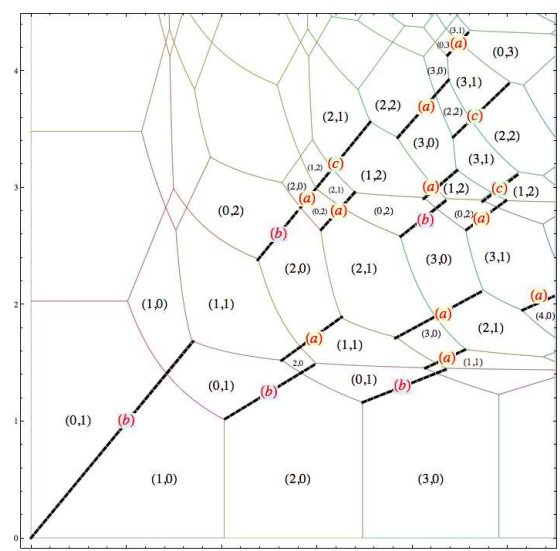

Figure 2. The critical index map for the 3D RayleighBenard convection with respect to the horizontal length scales $L_{1}$ and $L_{2}$, from [33].

1.1.3. The main assumptions on the nonlinear operator. We assume that $G$ consists of higher order terms in $u$, that is $G_{\lambda}(u)=o\left(\|u\|_{X_{\alpha}}\right)$ where $X_{\alpha}$ is an interpolation space with $0 \leq \alpha<1$. This ensures that (1) admits the homogeneous steady state solution

$$
u(t)=0, \quad \forall t \geq 0 .
$$

We consider the following Taylor expansion of $G$.

$$
G(u)=G_{2}(u, u)+G_{3}(u, u, u)+\cdots
$$

Here $G_{2}$ is the bilinear and $G_{3}$ is the trilinear operator of the Taylor expansion of $G$ and the rest of the expansion will not play a role in the analysis.

The main assumption is the following orthogonality conditions on the bilinear $G_{2}$ and trilinear $G_{3}$ parts of the nonlinear operator with respect the basis vectors $e_{i, j}$. We assume that if $\pm i_{r} \pm j_{r} \neq \pm k_{r}$ for some choice of \pm and at least one of $r=1,2$ then

$$
\left\langle G_{2}\left(e_{i_{1}, i_{2}}, e_{j_{1}, j_{2}}\right), e_{k_{1}, k_{2}}\right\rangle=0 .
$$

Here $\langle\cdot\rangle$ represent the inner product of $X$. Similarly, $\pm i_{r} \pm j_{r} \pm k_{r} \neq \pm l_{r}$ for some choice of \pm and at least one of $r=1,2$, we assume that

$$
\left\langle G_{3}\left(e_{i_{1}, i_{2}}, e_{j_{1}, j_{2}}, e_{k_{1}, k_{3}}\right), e_{l_{1}, l_{2}}\right\rangle=0 .
$$

Such orthogonality conditions are typical for trigonometric basis functions and nonlinear operators which are products of functions and their derivatives. Our main assumptions are satisfied in many physically interesting systems such as the convective motions of fluids [17, 34, 33, $10,11]$, reaction-diffusion systems [25, 19, 26, 42] and pattern formation equations $[6,41,5]$. We will also present several applications where these assumptions hold in Section 3. 
For example for modes given by (4) and a general nonlinear operator of the form

$$
G(u)=a_{1} u^{2}+a_{2} u u_{x}+a_{3} u u_{y}+a_{4} u^{3}+a_{5} u^{2} u_{x}+\cdots
$$

where $a_{i}$ are constants and the usual $L_{2}$ inner product, the assertions hold true due to the orthogonality of trigonometric functions.

1.2. Discussion of the main results. We first derive the general structure of the reduced (amplitude) equations by using the center manifold reduction. Letting $u_{1}(t) f_{1}+u_{2}(t) f_{2}$ to denote the center part of the solution, we obtain the following equations.

$$
\begin{array}{ll}
\frac{d u_{1}}{d t}=\beta(\lambda) u_{1}+a_{1} u_{1} u_{2} & +u_{1}\left(a_{2} u_{1}^{2}+a_{3} u_{2}^{2}\right)+O(4), \\
\frac{d u_{2}}{d t}=\beta(\lambda) u_{2}+b_{1} u_{1}^{2} & +u_{2}\left(b_{2} u_{1}^{2}+b_{3} u_{2}^{2}\right)+O(4) .
\end{array}
$$

These equations describe the long time behaviour of the system, near the transition point $\lambda=\lambda_{c}$ close to the basic steady state solution. The reduced equations consist of a quadratic part with coefficients $a_{1}, b_{1}$ due to the bilinear interactions between the critical modes and a cubic part with coefficients $a_{2}, a_{3}, b_{2}$ and $b_{3}$ due to the bilinear interactions of the critical modes with the higher frequency modes plus trilinear self interactions of the critical modes. Our analysis of the reduced equations shows that when none of the coefficients $a_{1}, b_{1}, b_{3}$ vanish, the type of transition depends only on these three parameters.

In this paper, we address the case $a_{1} \neq 0, b_{1} \neq 0$ and $b_{3} \neq 0$. In the case $a_{1}=b_{1}=0$, there are no bilinear interactions among the critical modes, and the behavior of the system is determined by the cubic coefficients $a_{2}, a_{3}, b_{2}, b_{3}$. That case is generic case when the first two critical modes are both roll-type or both rectangle-type and is also often encountered in the applications [33]. It also occurs under certain symmetry conditions which frequently arise in nonlinear systems of interest. We will address this case in a future study.

Next, by a detailed analysis of the reduced equations, we describe the bifurcated steady states and their stability and describe all the possible transition scenarios. Due to the interactions of these two modes, a variety of new states emerge after transition, including those associated with hexagonal patterns.

In terms of transition analysis, our guiding principle is the dynamic transition theory of Ma and Wang [23]. The key philosophy of dynamic transition theory is to search for the full set of transition states, giving a complete characterization of stability and transition. The set of transition states is a local attractor, representing the physical reality after the transition. As a general principle, dynamic transitions of all dissipative systems are classified into three categories: continuous (Type-I), 
catastrophic (Type-II), and random (Type-III). Intuitively, a continuous transition occurs when the system transitions to a nearby local attractor, a catastrophic transition occurs when there are no nearby local attractors after transition and finally random transition occurs when the system either moves to a local attractor or leave the local neighborhood depending on the initial perturbation. For some of the recent applications of this theory, we refer to [39, 40, 21, 15, 12].

Our analysis shows that four different transitions are possible depending on the signs of $a_{1} b_{1}$ and $b_{3}$. When $a_{1} b_{1}>0$ there are always bifurcated saddle mixed mode steady states near the basic solution on both sides of $\lambda=\lambda_{c}$. Moreover, the transition is either catastrophic or random depending on the sign of $b_{3}$. In the catastrophic transition, there are no steady states bifurcated from the basic solution after the transition $\lambda>\lambda_{c}$ and a repeller bifurcates on $\lambda<\lambda_{c}$. In the random transition scenario, the evolution of the system depends on the fluctuations (initial conditions) of the basic solution. Namely, the phase space separates into two sectorial regions where solutions starting from the first region leave the neighbourhood of the basic solution and solutions starting from the second region tend to an attractor nearby which consists of three steady states and the orbits between them.

When $a_{1} b_{1}<0$, the only bifurcated steady states are the two rolltype solutions which are symmetric of each other. Depending on the sign of $b_{3}$, there is either a continuous transition or a catastrophic transition. In the continuous transition case, the two roll type solutions are bifurcated on $\lambda>\lambda_{c}$ with one being stable and the other being saddle. In this case, there is an $S^{1}$ attractor which consists of these two steady states and the heteroclinic orbits connecting them. In the catastrophic transition case, there are no steady states bifurcated on $\lambda>\lambda_{c}$ and two roll type solutions are bifurcated on $\lambda<\lambda_{c}$ which form a repeller homeomorphic to $S^{1}$.

After the presentation of the general theory, we give three applications which show that all the transitions described by our main theorems are indeed observable. We consider two variants of the 2D Swift Hohenberg equation with quadratic-cubic nonlinearity. The last application is the 3D surface tension driven convection also known as Marangoni convection. For both systems, we demonstrate that our main assumptions are satisfied and describe the transition behavior from the main results we have proved.

1.3. Notations. We denote the adjoint eigenvectors by $f_{j}^{*}$ which are found by

$$
L^{*} f_{j}^{*}=\overline{\beta_{j}} f_{j}^{*},
$$

where $L^{*}$ is the adjoint linear operator. The eigenvectors of the linear and adjoint operator satisfy the orthogonality property

$$
\left\langle f_{j}, f_{k}^{*}\right\rangle=\delta_{j k}\left\langle f_{j}, f_{j}^{*}\right\rangle .
$$


with $\langle\cdot, \cdot\rangle$ denoting the inner product in $X$.

Recalling $G_{2}$ and $G_{3}$ from (7), for ease of notation, we will denote the bilinear and trilinear interactions of modes by

$$
\begin{aligned}
& G_{2}(i, j, k)=\frac{1}{\left\langle f_{k}, f_{k}^{*}\right\rangle}\left\langle G_{2}\left(f_{i}, f_{j}\right), f_{k}^{*}\right\rangle \\
& G_{2}^{s}(i, j, k)=G_{2}(i, j, k)+G_{2}(i, k, j)
\end{aligned}
$$

Similarly for the trilinear operator,

$$
\begin{aligned}
G_{3}(i, j, k, l) & =\frac{1}{\left\langle f_{l}, f_{l}^{*}\right\rangle}\left\langle G_{3}\left(f_{i}, f_{j}, f_{k}\right), f_{l}^{*}\right\rangle \\
G_{3}^{s}(i, j, k, l) & =\sum_{\sigma} G_{3}(\sigma(i, j, k), l)
\end{aligned}
$$

where the summation is over all permutations $\sigma$ of the triple $(i, j, k)$.

\section{Main tRAnsition THEOREMS}

In this section, we present the main results on transitions of the system (1) under the given conditions. According to dynamic transition theory, in any dissipative system, there are only three possible transition types. The intuitive understanding of three types of transitions is mentioned in the Introduction. For the exact definitions of three types of transitions we refer to [23].

2.1. The reduced equations. We first give the reduced equations with the exact expressions for their coefficients and mention several remarks regarding these equations.

We denote the center part of the solution spanned by the first two critical modes by

$$
u_{c}=u_{1}(t) f_{1}+u_{2}(t) f_{2}
$$

where $f_{1}$ and $f_{2}$ given by (3) are the first critical modes satisfying the PES conditions (2) and $u_{1}(t), u_{2}(t) \in \mathbb{R}$ are the time dependent amplitudes of these modes.

In Section 4, we prove that the dynamics of near the system near the origin and close to onset of transition $\lambda=\lambda_{c}$ is given by the following reduced equations become

$$
\begin{aligned}
& \frac{d u_{1}}{d t}=\beta(\lambda) u_{1}+F_{1}(x)+O(4) \\
& \frac{d u_{2}}{d t}=\beta(\lambda) u_{2}+F_{2}(x)+O(4)
\end{aligned}
$$

where the vector field $F=\left(F_{1}, F_{2}\right)$ is defined as follows:

$$
\begin{aligned}
& F_{1}\left(u_{1}, u_{2}\right)=a_{1} u_{1} u_{2}+u_{1}\left(a_{2} u_{1}^{2}+a_{3} u_{2}^{2}\right), \\
& F_{2}\left(u_{1}, u_{2}\right)=b_{1} u_{1}^{2}+u_{2}\left(b_{2} u_{1}^{2}+b_{3} u_{2}^{2}\right)
\end{aligned}
$$


and

$$
O(n)=O\left(|x|^{n}\right)+O\left(|x|^{n-1} \beta(\lambda)\right), \quad \text { as } x \rightarrow 0, \lambda \rightarrow \lambda_{c} .
$$

Here the coefficients of the quadratic terms are

$$
\begin{aligned}
& a_{1}=G_{2}^{s}(1,2,1) \\
& b_{1}=G_{2}(1,1,2)
\end{aligned}
$$

and the coefficients of the cubic terms are

$$
\begin{aligned}
a_{2}= & G_{3}(1,1,1,1)+\sum_{j \geq 3} \frac{-1}{\beta_{j}} G_{2}(1,1, j) G_{2}^{s}(1, j, 1) \\
a_{3}= & G_{3}^{s}(1,2,2,1)+\sum_{j \geq 3} \frac{-1}{\beta_{j}}\left(G_{2}(1,2, j) G_{2}^{s}(2, j, 1)\right. \\
& \left.+G_{2}(2,1, j) G_{2}^{s}(2, j, 1)+G_{2}(2,2, j) G_{2}^{s}(1, j, 1)\right) \\
b_{2}= & G_{3}^{s}(1,1,2,2)+\sum_{j \geq 3} \frac{-1}{\beta_{j}}\left(G_{2}(1,1, j) G_{2}^{s}(2, j, 2)\right. \\
& \left.+G_{2}(1,2, j) G_{2}^{s}(1, j, 2)+G_{2}(2,1, j) G_{2}^{s}(1, j, 2)\right) \\
b_{3}= & G_{3}(2,2,2,2)+\sum_{j \geq 3} \frac{-1}{\beta_{j}} G_{2}(2,2, j) G_{2}^{s}(2, j, 2)
\end{aligned}
$$

\section{Remarks.}

(1) We note that the cubic terms depend on the interactions of the first two modes with higher modes $\left(f_{j}\right.$ with $j \geq 3$ ), while the quadratic terms are determined solely by the self-interactions of the first two modes.

(2) The reduced equations (12) are symmetric under $u_{1} \mapsto-u_{1}$. As a result, the reduced phase portrait is symmetric with respect to $u_{2}$ axis.

(3) The first equation of the reduced equation can be solved as

$u_{1}(t)=u_{1}(0) \exp \left(\int_{0}^{t}\left(a_{1} u_{2}(s)+a_{2} u_{1}^{2}(s)+a_{3} u_{2}^{2}(s)\right) d s\right)$

As a result, in the reduced phase portrait, the sign of $u_{1}(0)$ is preserved by $u_{1}(t)$.

(4) When the first two modes are both rolls or both rectangles with equal wave numbers, our analysis can be extended to show that quadratic part of the reduced equations vanish while the cubic part remains the same. However, our main theorems only deal with the case of non-vanishing quadratic terms $a_{1}$ and $b_{1}$. We plan to address the case $a_{1}=b_{1}=0$ later.

(5) In this paper, we will always assume that $b_{3} \neq 0$. Otherwise, $(0,0)$ can easily seen to be non-isolated singular point of $F$. 
(6) When the critical modes have equal horizontal wave numbers as discussed in 1.1, we have the relation $k_{1}>j_{1}$ between the wave indices in (3). In that case, $C_{11} \cap C_{22}=\emptyset$ and the $G_{2}(2,2, j) G_{2}^{s}(1, j, 1)$ term in $a_{3}$ and $G_{2}(1,1, j) G_{2}^{s}(2, j, 2)$ in $b_{2}$ also vanish as a result.

Moreover, when the wave numbers of the critical modes are equal, in the sums (15), only modes $f_{j}$ spanned by $e_{i k}$ with wave indices $(i, k)$ in $C_{11}$ for $a_{2}, C_{12}$ for $a_{3}, b_{2}$ and $C_{22}$ for $b_{3}$ have to be considered where

$$
\begin{aligned}
& C_{11}=\left\{\left(2 j_{1}, 2 j_{2}\right),\left(2 j_{1}, 0\right),\left(0,2 j_{2}\right)\right\}, \\
& C_{12}=\left\{\left(k_{1}-j_{1}, j_{2}\right),\left(k_{1}+j_{1}, j_{2}\right)\right\}, \\
& C_{22}=\left\{\left(2 k_{1}, 0\right)\right\} .
\end{aligned}
$$

(7) The quadratic part of the reduced equations is degenerate and as a result, to study the transition behaviour, the cubic part (only $b_{3}$ term, as we shall see) is also necessary.

2.2. The statement of the main theorems. Before we state the main theorems, we briefly discuss the possible bifurcated steady state solutions of the system (1) and give the conditions which dictate their existence and stability.

$$
\begin{aligned}
& R_{1}=-\sqrt{-\frac{\beta}{b_{3}}} f_{2}+o(\sqrt{-\beta}), \\
& R_{2}=\sqrt{-\frac{\beta}{b_{3}}} f_{2}+o(\sqrt{-\beta})
\end{aligned}
$$

and

$$
\begin{aligned}
& H_{1}=\frac{1}{\sqrt{a_{1} b_{1}}} \beta f_{1}-\frac{1}{a_{1}} \beta f_{2}+o(\beta), \\
& H_{2}=-\frac{1}{\sqrt{a_{1} b_{1}}} \beta f_{1}-\frac{1}{a_{1}} \beta f_{2}+o(\beta),
\end{aligned}
$$

where $f_{1}, f_{2}$ are the first critical eigenmodes with eigenvalue $\beta$ satisfying the PES conditions given by (2). First, we note the scale difference between the steady states $R_{i}=O(-\sqrt{\beta})$ and $H_{i}=O(\beta)$ as $\beta \rightarrow 0$. Thus the steady states $H_{i}$ are much closer to the basic steady state than $R_{i}$. Second $R_{i}$ denote the bifurcated solutions which have the spatial roll pattern $e_{k_{1}, 0}$ plus small perturbations when the eigenmodes are given by (3). These solutions are defined only when $\beta(\lambda) b_{3}<0$. Third, $H_{i}$ denote the mixed mode bifurcated steady state solutions which may spatially represent hexagonal patterns. These solutions are defined when $a_{1} b_{1}>0$.

As is well known the transitions of the system are captured by the reduced equations (12) which contains 6 coefficients determined by the system. In the case $a_{1} \neq 0, b_{1} \neq 0$, the critical modes have self interactions. In addition, if $b_{3} \neq 0$, we will show that the transition of the 
system depends only on the signs of the 3 out of 6 coefficients $a_{1}, b_{1}$ and $b_{3}$ in (12). In this case, the remaining coefficients $a_{2}, a_{3}$ and $b_{2}$ play a quantitative but not a qualitative role. Our main theorems classify the transitions depending on the signs of these three coefficients as well as the stability of the above steady states. We summarize this in Table 1 and Table 2.

\begin{tabular}{|c|c|c|c|c|}
\hline$a_{1} b_{1}$ & $b_{3}$ & Theorem & Transition Type & Transition Diagram \\
\hline$>0$ & $<0$ & Theorem 2.1(i) & random & Figure 3 \\
$>0$ & $>0$ & Theorem 2.1(ii) & catastrophic & Figure 4 \\
$<0$ & $<0$ & Theorem 2.2(i) & continuous & Figure 5 \\
$<0$ & $>0$ & Theorem 2.1(ii) & catastrophic & Figure 6 \\
\hline
\end{tabular}

TABLE 1. The type of transition and the conditions required.

\begin{tabular}{|c|c|c|c|c|}
\hline$a_{1} b_{1}$ & $b_{3}$ & $H_{i}$ on $\lambda \neq \lambda_{c}$ & $R_{i}$ on $\lambda<\lambda_{c}$ & $R_{i}$ on $\lambda>\lambda_{c}$ \\
\hline$>0$ & $<0$ & both are SAD & DNE & $\begin{array}{l}R_{1} \text { is a } \mathrm{SN}, \\
R_{2} \text { is a } \mathrm{SAD}\end{array}$ \\
\hline$>0$ & $>0$ & both are SAD & $\begin{array}{l}R_{1} \text { is a } \mathrm{SAD}, \\
R_{2} \text { is an } \mathrm{UN}\end{array}$ & DNE \\
\hline$<0$ & $<0$ & DNE & DNE & $\begin{array}{l}R_{1} \text { is a } \mathrm{SN}, \\
R_{2} \text { is a SAD }\end{array}$ \\
\hline$<0$ & $>0$ & DNE & $\begin{array}{l}R_{1} \text { is a } \mathrm{SAD}, \\
R_{2} \text { is an } \mathrm{UN}\end{array}$ & DNE \\
\hline
\end{tabular}

TABLE 2. Stability properties of the bifurcated steady states for $a_{1}>0$. For $a_{1}<0$, the stability of $H_{i}$ is unchanged while the stability of $R_{1}$ and $R_{2}$ is exchanged. Here $\mathrm{SAD}=$ saddle, $\mathrm{SN}=$ stable node, $\mathrm{UN}=$ unstable node, $\mathrm{DNE}=$ does not exist.

Under the conditions given in Section 1.1, the transition behavior of the general dissipative system (1) is given by the following two theorems.

Theorem 2.1. Assume $a_{1} b_{1}>0$.

i) If $b_{3}<0$ then the system (1) undergoes a random (Type-III) transition at $\lambda=\lambda_{c}$ described as below. 
a) The topological structure of the transition is as in Figure 3.

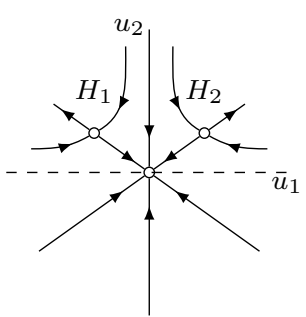

(a) $\lambda<\lambda_{c}$

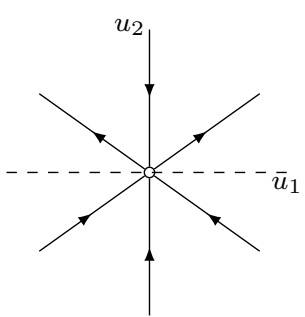

(b) $\lambda=\lambda_{c}$

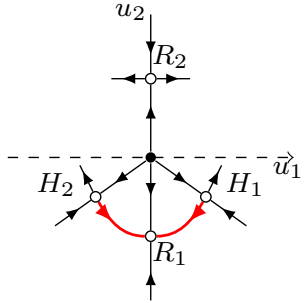

(c) $\lambda>\lambda_{c}$

Figure 3. The structure of the transition for $a_{1} b_{1}>0$, $b_{3}<0, a_{1}>0$. The bifurcated attractor $\Sigma_{\lambda}$ on $\lambda>\lambda_{c}$ is shown in red. When $a_{1}<0$, the assertions given by Theorem 2.1 hold true with the regions and the steady states flipped with respect to the $u_{1}$ axis.

b) There is a neighborhood $\mathcal{U}$ of $\phi=0$ in the phase space $X$ such that for any $\lambda_{c}<\lambda<\lambda_{c}+\epsilon$ with some $\epsilon>0, \mathcal{U}$ can be decomposed into two open sets $\mathcal{U}_{1}^{\lambda}, \mathcal{U}_{2}^{\lambda}$,

$$
\overline{\mathcal{U}}=\overline{\mathcal{U}_{1}^{\lambda}} \cup \overline{\mathcal{U}_{2}^{\lambda}}, \quad \mathcal{U}_{1}^{\lambda} \cap \mathcal{U}_{2}^{\lambda}=\emptyset
$$

such that

$$
\begin{array}{ll}
\lim _{\lambda \rightarrow \lambda_{c}} \limsup _{t \rightarrow \infty}\left\|S_{\lambda}(t, \varphi)\right\|_{X}=0 & \forall \varphi \in \mathcal{U}_{1}^{\lambda}, \\
\limsup _{t \rightarrow \infty}\left\|S_{\lambda}(t, \varphi)\right\|_{X} \geq \delta>0 & \forall \varphi \in \mathcal{U}_{2}^{\lambda},
\end{array}
$$

for some $\delta>0$. Here $S_{\lambda}$ is the evolution of the solution with initial data $\varphi$. Moreover $\mathcal{P}\left(U_{1}^{\lambda}\right), \mathcal{P}\left(U_{2}^{\lambda}\right)$ are sectorial regions as shown in Figure $3(c)$ with angles $\pi-2 \theta$ and $\pi+2 \theta$ respectively, where $\theta=\arctan \sqrt{a_{1} / b_{1}}$ and $\mathcal{P}$ is the projection onto the plane spanned by $f_{1}, f_{2}$.

c) The system bifurcates to an attractor $\Sigma_{\lambda}$ which consists of three steady states $R_{1}, H_{1}, H_{2}$ and the heteroclinic orbits connecting $H_{i}$ to $R_{1}, i=1,2$. Namely, $\Sigma_{\lambda}$ is the arc connecting these three steady states as shown in Figure 3(c), and has $\mathcal{U}_{1}^{\lambda}$ as its basin of attraction.

ii) If $b_{3}>0$ then the system undergoes a catastrophic (Type-II) transition at $\lambda=\lambda_{c}$ and the following assertions are true:

a) The topological structure of the transition is as given by Figure 4. 


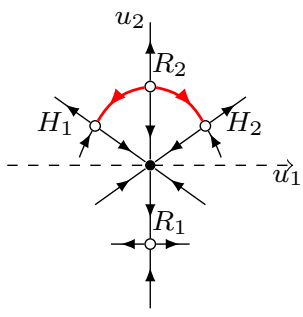

(a) $\lambda<\lambda_{c}$

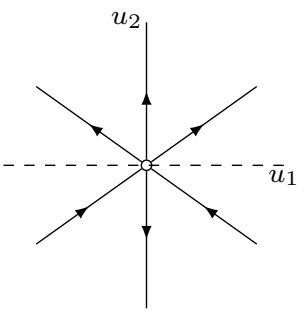

(b) $\lambda=\lambda_{c}$

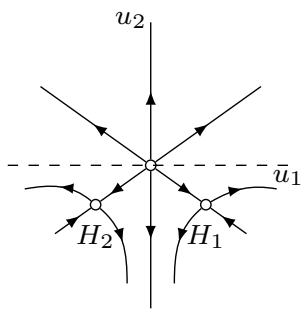

(c) $\lambda>\lambda_{c}$

FIgURE 4. The structure of transition for $a_{1} b_{1}>0, b_{3}>$ $0, a_{1}>0$. The bifurcated repeller $\Sigma_{\lambda}$ on $\lambda<\lambda_{c}$ is shown in red color. For $a_{1}<0$, the same transition diagram is obtained with $H_{1}, H_{2}$ solutions reflected along $u_{1}$ axis for $\lambda>\lambda_{c}$.

b) There is a bifurcated repeller $\Sigma_{\lambda}$ on $\lambda<\lambda_{c}$ which consists of three steady states, $\mathrm{H}_{1}, \mathrm{H}_{2}, \mathrm{R}_{2}$ and the heteroclinic orbits connecting $R_{2}$ to $H_{1}$ and $H_{2}$ respectively. $\Sigma_{\lambda}$, topologically.

c) Finally for $\lambda_{c}+\epsilon>\lambda>\lambda_{c}$ there is an open neighborhood $\mathcal{U}$ of $\phi=0$ and a dense, open subset $\mathcal{U}^{\lambda}$ of $\mathcal{U}$ such that

$$
\limsup _{t \rightarrow \infty}\left\|S_{\lambda}(t, \varphi)\right\|_{X} \geq \delta>0, \quad \forall \varphi \in \mathcal{U}^{\lambda}
$$

for some $\delta>0$.

Theorem 2.2. Assume $a_{1} b_{1}<0$.

i) If $b_{3}<0$, then the system (1) undergoes a continuous (Type-I) transition at $\lambda=\lambda_{c}$ described as below.

a) The topological structure is as given by Figure 5 .

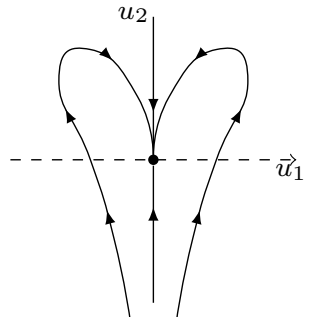

(a) $\lambda \leq \lambda_{c}$

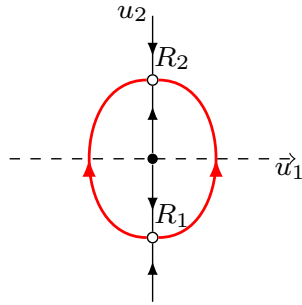

(b) $\lambda>\lambda_{c}$

FiguRE 5 . The structure of transition for $a_{1} b_{1}<0, b_{3}<0$.

b) The system bifurcates on $\lambda>\lambda_{c}$ to an attractor $\Sigma_{\lambda}$ which is homeomorphic to $S^{1} . \Sigma_{\lambda}$ consists of two singular points $R_{i}, i=1,2$ and two heteroclinic orbits connecting them. Moreover there exists a neighborhood $\mathcal{U}^{\lambda}$ of $\phi=0$ such 
that $\Sigma_{\lambda}$ attracts $\mathcal{U}^{\lambda} \backslash \Gamma$ where $\Gamma$ is the stable manifold of $\phi=0$ with $\operatorname{codim} \Gamma=2$.

ii) If $b_{3}>0$, then the system undergoes a catastrophic (Type-I) transition at $\lambda=\lambda_{c}$.

a) The topological structure is as given by Figure 6 .

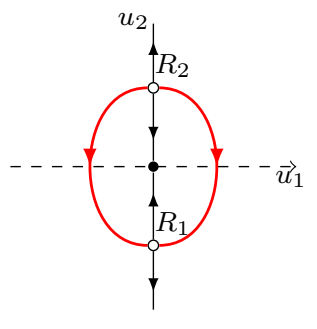

(a) $\lambda<\lambda_{c}$

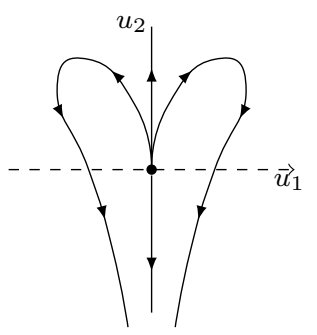

(b) $\lambda \geq \lambda_{c}$

FiguRE 6 . The structure of transition for $a_{1} b_{1}<0, b_{3}>0$.

b) The system bifurcates to a repeller $\Sigma_{\lambda}$ on $\lambda<\lambda_{c}$ which consists of two critical points $R_{i}, i=1,2$ and heteroclinic orbits connecting them.

\section{Applications}

In this section, we give several applications to demonstrate that all the results obtained in our main theorems are indeed realizable.

\subsection{D Swift-Hohenberg Equation with quadratic-cubic non-} linearity. We first consider the 2D Swift-Hohenberg equation

$$
u_{t}=\lambda u-(\Delta+k)^{2} u+\alpha_{2} u^{2}-\alpha_{3} u^{3}
$$

with $\alpha_{2}, \alpha_{3}, k, \lambda \in \mathbb{R}$ and $u=u(x, y, t)$ is the unknown function. The Swift-Hohenberg equation (SHE) was first proposed in 1977 [36] as a simple model for the Rayleigh-Benard instability of roll waves. For previous results on instabilities and physical aspects of the model, we refer to $[29,3,18,13]$

We consider a spatial domain

$$
\Omega=(0, \sqrt{3} \pi) \times(0, \pi),
$$

and Neumann boundary conditions

$$
\frac{\partial u}{\partial \nu}=\frac{\partial \Delta u}{\partial \nu}=0 \quad \text { on } \partial \Omega,
$$

where $\nu$ is the unit outward normal to $\partial \Omega$. We also assume zero mean conditions

$$
\iint_{\Omega} u d x d y=0 .
$$


For the functional setting, let

$$
\begin{aligned}
H & =\left\{u \in L^{2}(\Omega) \mid \int_{\Omega} u \mathrm{~d} x=0\right\}, \\
H_{1} & =\left\{u \in H^{4}(\Omega) \cap H \mid u \text { satisfies (21) and (22) }\right\} .
\end{aligned}
$$

Let $L_{\lambda}: H_{1} \rightarrow H$ and $G: H_{1} \rightarrow H$ be defined by

$$
\begin{aligned}
L_{\lambda} u & =\lambda u-(\Delta+k)^{2} u, \\
G(u) & =\alpha_{2} u^{2}-\alpha_{3} u^{3} .
\end{aligned}
$$

Then the problem (19) with (21) can be written as

$$
\begin{aligned}
& \frac{\mathrm{d} u}{\mathrm{~d} t}=L_{\lambda} u+G(u), \\
& u(0)=u_{0} .
\end{aligned}
$$

Under these conditions, the eigenvalue problem

$$
\lambda u-(\Delta+k)^{2} u=\beta u
$$

has eigenfunctions

$$
e_{j_{1}, j_{2}}(x, y)=\cos \left(j_{1} \frac{x}{\sqrt{3}}\right) \cos \left(j_{2} y\right)
$$

and eigenvalues

$$
\beta_{j_{1}, j_{2}}=\lambda-\left(k-|J|^{2}\right)^{2}, \quad J=\left(j_{1}, j_{2}\right) \in \mathcal{J}
$$

Here

$$
\begin{gathered}
\left|\left(j_{1}, j_{2}\right)\right|^{2}=\frac{j_{1}^{2}}{3}+j_{2}^{2} \\
\mathcal{J}=\left\{\left(j_{1}, j_{2}\right) \in \mathbb{Z}_{\geq 0} \times \mathbb{Z}_{\geq 0}:\left(j_{1}, j_{2}\right) \neq(0,0)\right\}
\end{gathered}
$$

It is easy to see that the PES condition is satisfied with

$$
\lambda_{c}(k)=\min _{J \in \mathcal{J}}\left(k-|J|^{2}\right)^{2}=\left(k-\left|J_{i}\right|^{2}\right)^{2}, \quad i=1, \ldots, n
$$

The minimum occurs at $|J|^{2}=k$ when $k \in \mathcal{J}$. If $k \notin \mathcal{J}$, then it occurs at one of $k_{1}, k_{2} \in \mathcal{J}$ for which $k_{1}<k<k_{2}$. Note that

$$
|(1,0)|<|(0,1)|<|(1,1)|=|(2,0)|<|(2,1)|<\cdots
$$

Thus solving $\left(k-|J|^{2}\right)^{2}=\left(k-|\tilde{J}|^{2}\right)^{2}$ for $k$ for the first two consecutive $|J|,|\tilde{J}|$ gives the following result.

$$
\begin{cases}J_{1}=(1,0) & \text { if } 0<k \leq 4 / 6 \\ J_{1}=(0,1) & \text { if } 4 / 6 \leq k \leq 7 / 6 \\ J_{1}=(1,1), J_{2}=(2,0) & \text { if } 7 / 6 \leq k \leq 11 / 6 \\ J_{1}=(2,1) & \text { if } 11 / 6 \leq k<19 / 6\end{cases}
$$

In particular, note that when

$$
7 / 6<k<11 / 6, \quad \lambda_{c}=(k-4 / 3)^{2},
$$



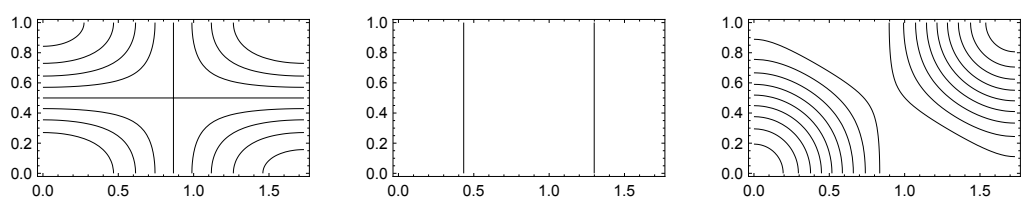

FiguRE 7. The bifurcated solutions.

there are two critical modes

$$
f_{1}=e_{1,1}=\cos \frac{x}{\sqrt{3}} \cos y, \quad f_{2}=e_{2,0}=\cos \frac{2 x}{\sqrt{3}},
$$

with corresponding eigenvalues satisfying the PES condition

$$
\begin{array}{r}
\beta_{1,1}=\beta_{2,0}=\lambda-\lambda_{c} \begin{cases}<0, & \lambda<\lambda_{c} \\
=0, & \lambda=\lambda_{c} \\
>0, & \lambda>\lambda_{c}\end{cases} \\
\beta_{j_{1}, j_{2}}<0, \quad\left(j_{1}, j_{2}\right) \notin\{(1,1),(2,0)\} .
\end{array}
$$

Thus the main assumptions are all satisfied. Hence, the PDE system can be reduced to the ODE system (12) near $\lambda=\lambda_{c}$ and for small $\left(u_{1}, u_{2}\right)$ where $u_{1}, u_{2}$ are the time dependent amplitudes of the modes $f_{1}, f_{2}$.

Now we can compute the coefficients (14), (15) that determines the type of transition. We let

Hence

$$
\begin{aligned}
G_{2}(i, j, k) & =\alpha_{2} \int_{\Omega} f_{i} f_{j} f_{k} d x d y \\
G_{3}(i, j, k, l) & =\alpha_{3} \int_{\Omega} f_{i} f_{j} f_{k} f_{l} d x d y
\end{aligned}
$$

$$
a_{1}=\alpha_{2}, \quad b_{1}=\frac{\alpha_{2}}{4}, \quad b_{3}=\frac{3\left(\alpha_{2}^{2}+4(3 k-10) \alpha_{3}\right)}{(160-48 k)} .
$$

Note that $a_{1} b_{1} \geq 0$. Also if $\alpha_{2} \neq 0$ then necessarily $a_{1} b_{1}>0$. For the range of $k$ values we are interested, $7 / 6<k<11 / 6$,

$$
b_{3} \sim \alpha_{2}^{2}+4(3 k-10) \alpha_{3}
$$

and $b_{3}>0$ if $\alpha_{3}<0$ and can be of both signs when $\alpha_{3}>0$.

Theorem 3.1. Under the conditions (26) and $\alpha_{2} \neq 0$, the basic solution $u=0$ of the equation (25) undergoes a transition at $\lambda=\lambda_{c}$. The type of transition is either continuous if $b_{3}<0$ or catastrophic if $b_{3}>0$ as described by Theorem 2.1. In particular, if $\alpha_{3} \leq 0$ then $b_{3}>0$, while for $\alpha_{3}>0$, depending on $\alpha_{2}, b_{3}$ can be of both signs, where $\alpha_{2}$ and $\alpha_{3}$ are the coefficients of the bilinear and trilinear terms in (19). The structure of the bifurcated mixed modes are as given in Figure $\%$ 
3.2. Modified 2D Swift Hohenberg Equation. We will now show that both transition scenarios described by Theorem 2.2 are possible. For this let us consider the following equation which is more general than (19).

$$
u_{t}=\lambda u-(\Delta+k)^{2} u+G(u, u)-\alpha_{3} u^{3}
$$

where the bilinear operator is

$$
G(u, v)=\left(c_{1}+c_{2} u_{x}+c_{3} u_{y}\right) v+u\left(c_{4} v_{x}+c_{5} v_{y}\right)
$$

on the rectangular domain (20) with the following boundary conditions

$$
\begin{aligned}
& u=\Delta u=0, \quad x=0, \sqrt{3} \pi, \\
& u_{y}=\Delta u_{y}=0, \quad y=0, \pi .
\end{aligned}
$$

This time, the basis functions are

$$
\begin{gathered}
e_{j_{1}, j_{2}}(x, y)=\sin \left(j_{1} \frac{x}{\sqrt{3}}\right) \cos \left(j_{2} y\right) \\
5 / 6<k<11 / 6, \quad \lambda_{c}=(k-4 / 3)^{2},
\end{gathered}
$$

and there are two critical modes

$$
f_{1}=e_{1,1}=\sin \frac{x}{\sqrt{3}} \cos y, \quad f_{2}=e_{2,0}=\sin \frac{2 x}{\sqrt{3}},
$$

with corresponding eigenvalues satisfying the PES condition (27). In this case, the numbers describing the transition are found to be

$a_{1}=-\frac{\left(c_{2}+c_{4}\right)^{2}}{2 \sqrt{3}}, \quad b_{1}=\frac{c_{2}+c_{4}}{4 \sqrt{3}}, \quad b_{3}=\frac{\left(c_{2}+c_{4}\right)^{2}+6(10-3 k) \alpha_{3}}{24 k-80}$

It is readily seen that for $c_{2}+c_{4} \neq 0, a_{1} b_{1}<0$ and the transition is described by the following theorem.

Theorem 3.2. Under the conditions (31) and $c_{2}+c_{4} \neq 0$, the basic solution $u=0$ of the equation (28) with (30) undergoes a transition at $\lambda=\lambda_{c}$. The type of transition is either continuous if $b_{3}<0$ or catastrophic if $b_{3}>0$ as described by Theorem 2.2. In particular, if $\alpha_{3} \geq 0$ then $b_{3}<0$, while for $\alpha_{3}<0$, depending on $c_{2}+c_{4}, b_{3}$ can be of both signs, where $c_{2}, c_{4}$ and $\alpha_{3}$ are the coefficients of the bilinear and trilinear terms in (28).

3.3. Surface tension driven convection. In this section, we present known results on the hexagonal pattern formation in surface tension driven convection also known as Marangoni convection to show that it fits into the framework we present in this study. The nondimensional form of the equations describing the Marangoni convection without 
gravity are, [8, 38],

$$
\begin{aligned}
& \frac{\partial \mathbf{u}}{\partial t}+(\mathbf{u} \cdot \nabla) \mathbf{u}=\operatorname{Pr}(-\nabla p+\Delta \mathbf{u}) \\
& \frac{\partial \theta}{\partial t}+(\mathbf{u} \cdot \nabla) \theta=w+\Delta \theta \\
& \nabla \cdot \mathbf{u}=0
\end{aligned}
$$

For the physical description and results about previous results on the instabilities of the above system, we refer to [27, 30, 31, 8, 7, 9, 4, 10]. Here $\mathbf{u}=(u, v, w)$ is the velocity field, $T$ is the temperature, $p$ is the pressure, $\operatorname{Pr}=\nu / \kappa>0$ is the Prandtl number. The unknowns represent a deviation from the motionless basic solution with a linear temperature profile given by,

$$
\begin{aligned}
& \mathbf{u}_{b}=0 \\
& T_{b}=T_{0}+\left(T_{1}-T_{0}\right) z \\
& p_{b}(z)=p_{0}+g \rho_{0}\left(z-\alpha\left(T_{1}-T_{0}\right) z^{2} / 2\right)
\end{aligned}
$$

where $T_{0}, T_{1}$ are the temperatures at $z=0$ and $z=1$ respectively.

We consider the equations (1) on a rectangular domain

$$
\Omega=\left(0, L_{1}\right) \times\left(0, L_{2}\right) \times(0,1) \subset \mathbb{R}^{3} .
$$

We supplement the above system with free-slip boundary conditions on the lateral boundaries, and the rigid (no slip) boundary condition and perfectly conducting on the bottom boundary. The top surface is assumed to be a non-deformable free surface with a surface tension of the form

$$
\xi=\xi_{0}\left(1-\gamma_{T} \theta\right)
$$

Namely, the boundary conditions are as follows:

$$
\begin{array}{ll}
u=\frac{\partial v}{\partial x}=\frac{\partial w}{\partial x}=\frac{\partial \theta}{\partial x}=0 & \text { at } x=0, L_{1}, \\
\frac{\partial u}{\partial y}=v=\frac{\partial w}{\partial y}=\frac{\partial \theta}{\partial y}=0 & \text { at } y=0, L_{2}, \\
u=v=w=\theta=0 & \text { at } z=0, \\
\frac{\partial(u, v)}{\partial z}+\lambda \nabla_{H} \theta=w=\frac{\partial \theta}{\partial z}+\operatorname{Bi} \theta=0 & \text { at } z=1,
\end{array}
$$

where $\nabla_{H}=\left(\partial_{x}, \partial_{y}\right), \mathrm{Bi} \geq 0$ is the Biot number, and the Marangoni number $\lambda$ is the control parameter which represents the ratio of the destabilizing surface tension gradient to the stabilizing forces associated with thermal and viscous diffusion. 
By the separation of variables, we represent the solutions in the following form:

$$
\begin{aligned}
& u_{I}=U_{I}(z) \sin L_{1}^{-1} I_{x} \pi x \cos L_{2}^{-1} I_{y} \pi y, \\
& v_{I}=V_{I}(z) \cos L_{1}^{-1} I_{x} \pi x \sin L_{2}^{-1} I_{y} \pi y, \\
& w_{I}=W_{I}(z) \cos L_{1}^{-1} I_{x} \pi x \cos L_{2}^{-1} I_{y} \pi y, \\
& \theta_{I}=\Theta_{I}(z) \cos L_{1}^{-1} I_{x} \pi x \cos L_{2}^{-1} I_{y} \pi y,
\end{aligned}
$$

for $I=\left(I_{x}, I_{y}\right) \in \mathbb{Z} \times \mathbb{Z}$. If instead of free-slip boundaries, no-slip boundaries are considered then the corresponding eigenvalue problem has to be solved by numerical methods [7, 32].

In [10], the following are proved:

(1) When the relation (6) between the horizontal length scales of the domain is satisfied, it is possible that two modes with indices $\left(j_{1}, j_{2}\right)$ and $\left(0,2 j_{2}\right)$ are the first two critical modes satisfying the PES condition (2) at the critical Marangoni number $\lambda_{c}$, introduced in [27] is defined as

$$
\lambda_{c}=\min _{\alpha} \frac{8 \alpha(\alpha \cosh \alpha+\operatorname{Bi} \sinh \alpha)(\alpha-\cosh \alpha \sinh \alpha)}{\alpha^{3} \cosh \alpha-\sinh ^{3} \alpha},
$$

where the minimum is taken over wave numbers

$$
\alpha=\alpha_{j, k}=\left(\left(L_{1}^{-1} j\right)^{2}+\left(L_{2}^{-1} k\right)\right)^{1 / 2} \pi .
$$

The map of critical index selection is similar to (2).

(2) Under the above setting, the system has a transition described by the reduced equations (12). Moreover the coefficients of the reduced equations satisfy

$$
a_{1}=4 b_{1}, \quad a_{3}=2 b_{3}, \quad 4 a_{2}=a_{3}+b_{2}
$$

(3) Thus $a_{1} b_{1}>0$ and the transition is as described by Theorem 2.1. The sign of $b_{3}$ is found to be negative in a limited parameter space and thus the transition is found to be random (Type-III).

\section{Proofs}

In this section, we give the proofs of our main theorems. Our strategy is first to obtain the reduction onto the center manifold of the dynamics and second, to analyze the reduced equations.

4.1. Center manifold approximation. We recall that the center part of the solution is given by

$$
u_{c}=u_{1}(t) f_{1}+u_{2}(t) f_{2}
$$

The rest of the solution is approximated by the center manifold which we expand as

$$
\Phi=\sum_{k \geq 3} \Phi_{k}(t) f_{k}
$$


As is well known, see [23], the lowest order (quadratic) approximation of $\Phi$ is obtained as the solution of

$$
\mathcal{L} \Phi=-P_{s} G\left(u_{c}\right)+o(2) .
$$

Here $P_{s}: X \rightarrow E_{s}$ is the projection operator where $E_{s}$ is the stable space which is the span of $\left\{f_{j}: j \geq 3\right\}$ in $X$ and $\mathcal{L}=\left.L\right|_{E_{s}}$ is the restriction of the linear operator onto the stable space.

Since $f_{j} \in E_{s}$, for $j \geq 3$, we have

$$
\left\langle P_{s}(\cdot), f_{j}^{*}\right\rangle=\left\langle\cdot, f_{j}^{*}\right\rangle, \quad j \geq 3 .
$$

Plugging (35) in (36), taking the inner product of (36) with $f_{j}, j \geq 3$ and making use of (37), we get

$$
\Phi_{j} \beta_{j}\left\langle f_{j}, f_{j}^{*}\right\rangle=\left\langle\Phi, L^{*} f_{j}\right\rangle=-\left\langle G\left(u_{c}\right), f_{j}^{*}\right\rangle, \quad j \geq 3 .
$$

From (38), using the notation (10), we obtain the below formula for the coefficients of the center manifold.

$$
\begin{aligned}
\Phi_{j} & =\frac{-1}{\beta_{j}\left\langle f_{j}, f_{j}^{*}\right\rangle}\left\langle G\left(u_{c}\right), f_{j}^{*}\right\rangle \\
& =\frac{-1}{\beta_{j}} \sum_{m, n=1,2} u_{m} u_{n} G_{2}(m, n, j), \quad j \geq 3 .
\end{aligned}
$$

Notice that $\Phi_{j}$ is independent of the trilinear operator $G_{3}$.

Now the reduced equations of the system are obtained by plugging

$$
u=u_{c}+\Phi
$$

into the main equation (1), which is basically considering the dynamics on the center manifold and taking projection onto the center space, that is the span of the $\left\{f_{1}, f_{2}\right\}$. This gives

$$
\frac{d}{d t}\left\langle u, f_{k}^{*}\right\rangle=\left\langle L_{\lambda} u, f_{k}^{*}\right\rangle+\left\langle G_{\lambda}(u), f_{k}^{*}\right\rangle, \quad k=1,2,
$$

which is equivalent to the system

$$
\frac{d u_{k}}{d t}=\beta_{k} u_{k}+\frac{1}{\left\langle f_{k}, f_{k}^{*}\right\rangle}\left\langle G\left(u_{c}+\Phi\right), f_{k}^{*}\right\rangle, \quad k=1,2 .
$$

To obtain a closed system, we have to write the nonlinear term above as a function of $u_{1}, u_{2}$. This can be obtained as follows

$$
\begin{aligned}
& \frac{1}{\left\langle f_{k}, f_{k}^{*}\right\rangle}\left\langle G\left(u_{c}+\Phi\right), f_{k}^{*}\right\rangle \\
& =\frac{1}{\left\langle f_{k}, f_{k}^{*}\right\rangle}\left\langle G_{2}\left(u_{c}, u_{c}\right)+G_{2}^{s}\left(u_{c}, \Phi\right)+G_{3}\left(u_{c}, u_{c}, u_{c}\right), f_{k}^{*}\right\rangle+O(4) \\
& :=P_{2, k}+P_{3, k}+O(4), \quad k=1,2
\end{aligned}
$$

and (40) becomes

$$
\frac{d u_{k}}{d t}=\beta_{k} u_{k}+P_{2, k}\left(u_{1}, u_{2}\right)+P_{3, k}\left(u_{1}, u_{2}\right)+O(4), \quad k=1,2 .
$$


where $P_{2, k}\left(u_{1}, u_{2}\right)$ denote the quadratic terms in $u_{1}, u_{2}$ given by

$$
\begin{aligned}
P_{2, k} & =\frac{1}{\left\langle f_{k}, f_{k}^{*}\right\rangle}\left\langle G_{2}\left(u_{c}, u_{c}\right), f_{k}^{*}\right\rangle \\
& =\frac{1}{\left\langle f_{k}, f_{k}^{*}\right\rangle} \sum_{i, j=1}^{2}\left\langle G_{2}\left(u_{i} f_{i}, u_{j} f_{j}\right), f_{k}^{*}\right\rangle \\
& =\sum_{i, j=1}^{2} u_{i} u_{j} G_{2}(i, j, k), \quad k=1,2
\end{aligned}
$$

and $P_{3, k}\left(u_{1}, u_{2}\right), k=1,2$ denote the cubic terms given by

$$
\begin{aligned}
P_{3, k}= & \frac{1}{\left\langle f_{k}, f_{k}^{*}\right\rangle}\left\langle G_{2}^{s}\left(u_{c}, \Phi\right)+G_{3}\left(u_{c}, u_{c}, u_{c}\right), f_{k}^{*}\right\rangle \\
= & \frac{1}{\left\langle f_{k}, f_{k}^{*}\right\rangle} \sum_{i, l, m \in\{1,2\}, j \geq 3}\left\langle G_{2}^{s}\left(u_{i} f_{i}, \Phi_{j} f_{j}\right)+G_{3}\left(u_{i} f_{i}, u_{m} f_{m}, u_{n} f_{n}\right), f_{k}^{*}\right\rangle \\
= & \sum_{m, n \in\{1,2\}, j \geq 3} u_{i} u_{m} u_{n} \frac{-1}{\beta_{j}} G_{2}(m, n, j) G_{2}^{s}(i, j, k) \\
& \quad+\sum_{i, m, n \in\{1,2\}} u_{i} u_{m} u_{n} G_{3}(i, m, n, k)
\end{aligned}
$$

Now, we take into account the structure (3) of the eigenmodes into account.

4.2. Consequences of assumption on nonlinear operator. The condition (8) has the following implications.

$$
\begin{aligned}
& \left(l_{1}, l_{2}\right) \notin C_{11} \Longrightarrow\left\langle\left(G_{2}\left(e_{j_{1}, j_{2}}, e_{j_{1}, j_{2}}\right), e_{l_{1}, l_{2}}\right)\right\rangle=0 \\
& \left(l_{1}, l_{2}\right) \notin C_{12} \Longrightarrow\left\langle\left(G_{2}\left(e_{j_{1}, j_{2}}, e_{k_{1}, 0}\right), e_{l_{1}, l_{2}}\right)\right\rangle=0 \\
& \left(l_{1}, l_{2}\right) \notin C_{12} \Longrightarrow\left\langle\left(G_{2}\left(e_{k_{1}, 0}, e_{j_{1}, j_{2}}\right), e_{l_{1}, l_{2}}\right)\right\rangle=0 \\
& \left(l_{1}, l_{2}\right) \notin C_{22} \Longrightarrow\left\langle\left(G_{2}\left(e_{k_{1}, 0}, e_{k_{1}, 0}\right), e_{l_{1}, l_{2}}\right)\right\rangle=0
\end{aligned}
$$

where the index sets are as defined in (16).

Using the notation (10), the observations in (42)-(45) lead to the following observations

$$
\begin{gathered}
G_{2}(1,1,1)=G_{2}(2,2,1)=0 . \\
G_{2}(2,2,2)=G_{2}(2,1,2)=G_{2}(1,2,2)=0 .
\end{gathered}
$$

But it is possible that

$$
G_{2}(1,2,1) \neq 0, \quad G_{2}(2,1,1) \neq 0, \quad G_{2}(1,1,2) \neq 0
$$

By (16), we note that

$$
C_{11} \cap C_{12}=C_{12} \cap C_{22}=\emptyset
$$


which implies that the following products of nonlinear interactions of the critical modes with higher modes vanish.

$$
\begin{array}{ll}
G_{2}\left(\sigma_{1}(1,1, j)\right) G_{2}\left(\sigma_{2}(1,2, j)\right)=0, & \forall j \geq 3, \text { since } C_{11} \cap C_{12}=\emptyset \\
G_{2}\left(\sigma_{1}(1,2, j)\right) G_{2}\left(\sigma_{2}(2,2, j)\right)=0, & \forall j \geq 3, \text { since } C_{12} \cap C_{22}=\emptyset
\end{array}
$$

where $\sigma_{1}, \sigma_{2}$ are any permutations of the set $\{1,2,3\}$. For example, the first condition above is equivalent to the vanishing of the 18 products given by

$$
\begin{aligned}
& G_{2}(1,1, j) G_{2}(1,2, j)=G_{2}(1, j, 1) G_{2}(1,2, j)=G_{2}(j, 1,1) G_{2}(1,2, j)=0, \\
& G_{2}(1,1, j) G_{2}(1, j, 2)=G_{2}(1, j, 1) G_{2}(1, j, 2)=G_{2}(j, 1,1) G_{2}(1, j, 2)=0,
\end{aligned}
$$

and the same for the remaining 12 products as well for all $j \geq 3$.

Similarly, for the trilinear term, for $i, j, k, l \in\{1,2\}$, $(50)$

$i+j+k+l=1 \quad \bmod 2 \Longrightarrow G_{3}(i, j, k, l)=0 \Longrightarrow G_{3}^{s}(i, j, k, l)=0$.

4.3. Structure of the quadratic polynomials $P_{2, k}$. As a consequence of (47)

$$
G_{2}^{s}(1,2,2)=G(1,2,2)+G(2,1,2)=0 .
$$

Hence by (46), (47), and (51), the quadratic terms become

$$
\begin{aligned}
P_{2,1} & =u_{1}^{2} G(1,1,1)+u_{1} u_{2} G_{2}^{s}(1,2,1)+u_{2}^{2} G(2,2,1) \\
& =u_{1} u_{2} G_{2}^{s}(1,2,1) \\
P_{2,2} & =u_{1}^{2} G(1,1,2)+u_{1} u_{2} G_{2}^{s}(1,2,2)+u_{2}^{2} G(2,2,2) \\
& =u_{1}^{2} G(1,1,2)
\end{aligned}
$$

4.4. Structure of the cubic polynomials $P_{3, k}$. The coefficients of the following cubic terms vanish by (49) and (50).

(1) The term $u_{1}^{2} u_{2}$ in $P_{3,1}$

$$
\begin{gathered}
G_{3}^{s}(1,1,2,1)+\sum_{j \geq 3} \frac{-1}{\beta_{j}}\left(G(1,2, j) G_{2}^{s}(1, j, 1)+G(2,1, j) G_{2}^{s}(1, j, 1)\right. \\
\left.+G(1,1, j) G_{2}^{s}(2, j, 1)\right)=0,
\end{gathered}
$$

(2) The term $u_{2}^{3}$ in $P_{3,1}$

$$
G_{3}(2,2,2,1)+\sum_{j \geq 3} \frac{-1}{\beta_{j}} G(2,2, j) G_{2}^{s}(2, j, 1)=0,
$$

(3) The term $u_{1} u_{2}^{2}$ in $P_{3,2}$

$$
\begin{gathered}
G_{3}^{s}(1,2,2,2)+\sum_{j \geq 3} \frac{-1}{\beta_{j}}\left(G(1,2, j) G_{2}^{s}(2, j, 2)+G(2,1, j) G_{2}^{s}(2, j, 2)\right. \\
\left.+G(2,2, j) G_{2}^{s}(1, j, 2)\right)+=0,
\end{gathered}
$$


(4) The term $u_{1}^{3}$ in $P_{3,2}$

$$
G_{3}(1,1,1,2)+\sum_{j \geq 3} \frac{-1}{\beta_{j}}\left(G(1,1, j) G_{2}^{s}(1, j, 2)\right)=0,
$$

4.5. The existence of bifurcated steady state solutions. We start by finding the straight line orbits of the vector field $\left(F_{1}, F_{2}\right)$ given by (13) near $\left(u_{1}, u_{2}\right)=(0,0)$. Clearly $u_{1}=0$ is always a straight line orbit that is $d u_{1} / d t=0$. On $u_{1}=0$, the dynamics of $u_{2}$ is as follows.

$$
\frac{d u_{2}}{d t}=u_{2}\left(\beta+b_{3} u_{2}^{2}\right)+o\left(u_{2}^{4}\right) .
$$

For $\beta b_{3}<0$, we find the two bifurcated steady state solutions (roll pattern solutions) $R_{1}, R_{2}$ given by (17), that is with amplitudes $u_{1}=0$, and

$$
u_{1}=0, \quad u_{2}= \pm \sqrt{-\frac{\beta}{b_{3}}}+o(\sqrt{-\beta}) .
$$

Now we look for other straight line orbits $u_{1}=k u_{2}, k \neq 0$ near the origin. On such a straight line orbit, the following relation is satisfied

$$
k=\frac{u_{1}}{u_{2}}=\frac{F_{1}}{F_{2}}=\frac{a_{1} k u_{2}^{2}+O\left(u_{2}^{3}\right)}{b_{1} k^{2} u_{2}^{2}+O\left(u_{2}^{3}\right)}, \quad u_{2} \rightarrow 0 .
$$

Hence, for $k \neq 0, k$ should satisfy the relation

$$
k^{2} b_{1}=a_{1} .
$$

In particular, if $a_{1} b_{1}<0$, no such $k \neq 0$ exists.

On the other hand for $a_{1} b_{1}>0$, in addition to $u_{1}=0$, four more straight line orbits appear

$$
u_{1}=k_{i} u_{2}, \quad k_{i}=(-1)^{i} \sqrt{a_{1} / b_{1}}, \quad i=1,2 .
$$

The flow on the straight line $u_{1}=k_{i} u_{2}$ is given by

$$
\frac{d u_{2}}{d t}=u_{2}\left(\beta+b_{1} k_{i}^{2} u_{2}+\left(b_{2} k_{i}^{2}+b_{3}\right) u_{2}^{2}\right),
$$

which has two steady state solutions

$$
\begin{aligned}
& u_{1}=k_{i} u_{2}=(-1)^{i+1} \frac{\beta}{\sqrt{a_{1} b_{1}}}+O\left(\beta^{2}\right), \\
& u_{2}=-\frac{\beta}{b_{1} k_{i}^{2}}+O\left(\beta^{2}\right)=-\frac{\beta}{a_{1}}+O\left(\beta^{2}\right), \quad i=1,2 .
\end{aligned}
$$

On each line $u_{1}=k_{i} u_{2}, i=1,2$, there is a bifurcated steady state solution which gives the amplitudes of (hexagonal pattern solution) $H_{1}$ and $H_{2}$ given by (18). 
4.6. The stability of the bifurcated steady state solutions. The Jacobian matrix of the right hand side of the reduced equations (12) be

$$
D F\left(u_{1}, u_{2}\right)=\left(\begin{array}{cc}
3 a_{2} u_{1}^{2}+a_{3} u_{2}^{2}+a_{1} u_{2}+\beta & u_{1}\left(2 a_{3} u_{2}+a_{1}\right) \\
2 u_{1}\left(b_{2} u_{2}+b_{1}\right) & b_{2} u_{1}^{2}+3 b_{3} u_{2}^{2}+\beta
\end{array}\right) .
$$

Let us also denote the eigenvalues of $D F$ by $\lambda_{1}, \lambda_{2}$. We find that the eigenvalues of $D F$ at the steady states (55) as below. For $R_{1}$, that is for $\left(u_{1}, u_{2}\right)=\left(0,-\sqrt{-\frac{\beta}{b_{3}}}\right)+o(\sqrt{-\beta})$, they are

$$
\lambda_{1}=-2 \beta, \quad \lambda_{2}=-a_{1} \sqrt{-\frac{\beta}{b_{3}}}+O(\beta),
$$

and for $R_{2}$, that is for $\left(u_{1}, u_{2}\right)=\left(0, \sqrt{-\frac{\beta}{b_{3}}}\right)+o(\sqrt{-\beta})$, they are

$$
\lambda_{1}=-2 \beta, \quad \lambda_{2}=a_{1} \sqrt{-\frac{\beta}{b_{3}}}+O(\beta) .
$$

Thus the stability of the bifurcated solutions $R_{1}, R_{2}$ given by (17) for $\beta>0$ are as follows.

$$
\begin{array}{ll}
a_{1}>0 & R_{1} \text { is a stable node, } R_{2} \text { is a saddle } \\
a_{1}<0 & R_{1} \text { is a saddle, } R_{2} \text { is a stable node }
\end{array}
$$

Since the amplitude (57) of the mixed mode steady states are $u_{1}=$ $(-1)^{i+1} \frac{\beta}{\sqrt{a_{1} b_{1}}}+O\left(\beta^{2}\right), u_{2}=-\frac{\beta}{a_{1}}+O\left(\beta^{2}\right)$, we find that the Jacobian matrix of the mixed modes is

$$
\left(\begin{array}{cc}
\beta+a_{1} u_{2}+O\left(\beta^{2}\right) & a_{1} u_{1}+O\left(\beta^{2}\right) \\
2 b_{1} u_{1}+O\left(\beta^{2}\right) & \beta+O\left(\beta^{2}\right)
\end{array}\right)
$$

and the corresponding eigenvalues satisfy

$$
\lambda_{1}+\lambda_{2}=\beta+O\left(\beta^{2}\right)
$$

and

$$
\lambda_{1} \lambda_{2}=-2 a_{1} b_{1} u_{1} u_{2}+O\left(\beta^{3}\right)=-2 \beta^{2}+O\left(\beta^{3}\right)
$$

Thus we find the eigenvalues and the corresponding eigenvectors of the amplitudes of the equilibria $H_{i}$ as

$H_{1}: \quad \lambda_{1}=-\beta+O\left(\beta^{2}\right), v_{1}=\left(\begin{array}{c}-\frac{a_{1}}{\sqrt{a_{1} b_{1}}} \\ 1\end{array}\right), \quad \lambda_{2}=2 \beta+O\left(\beta^{2}\right), v_{2}=\left(\begin{array}{c}\frac{a_{1}}{2 \sqrt{a_{1} b_{1}}} \\ 1\end{array}\right)$

and

$H_{2}: \quad \lambda_{1}=-\beta+O\left(\beta^{2}\right), v_{1}=\left(\begin{array}{c}\frac{a_{1}}{\sqrt{a_{1} b_{1}}} \\ 1\end{array}\right), \quad \lambda_{2}=2 \beta+O\left(\beta^{2}\right), v_{2}=\left(\begin{array}{c}-\frac{a_{1}}{2 \sqrt{a_{1} b_{1}}} \\ 1\end{array}\right)$ 
Since the eigenvalues have always opposite signs near $\beta=0$, the equilibria $H_{i}$ given by (18) are always saddles whenever they exist.

The details of the proof of the main theorems follow from the above analysis and the dynamic transition theory [23, 22].

\section{SUMmarY AND DISCUSSION}

In this paper, we have found all possible transition scenarios of a general dissipative system with two dimensional critical center space. We have two main assumptions. First is the one that the one of the eigenmodes has a rectangle pattern and the other one has a roll pattern which is possible in spatial domains of at least two dimensions with homogeneous boundary conditions. The second one is an orthogonality condition on the nonlinear operator with respect to the basis vectors. This condition is generally satisfied when the bilinear and trilinear terms of the Taylor expansion of the nonlinear operator is a product of the unknown function and its derivatives.

Under the above general conditions, we manage to show that all three types of transitions of the dynamic transition theory are possible at the first criticality $\lambda_{c}$ under generic conditions. We also determine the bifurcated steady state solutions with their stability and the bifurcated attractors in each case. Finally, we give several applications to demonstrate that all the conclusions of our main theorems are observable.

Up until now, the dynamic transition theory has been used as a general tool to understand specific problems. This paper points in the direction of determination of transitions and pattern formations for a general class of problems. Thus we believe that the results presented in this study will provide a general framework for the determination and the validity of transitions in many applications.

\section{REFERENCES}

[1] Henri Bénard. Les tourbillons cellulaires dans une nappe liquide.-Méthodes optiques d'observation et d'enregistrement. Journal de Physique Théorique et Appliquée, 10(1):254-266, 1901.

[2] Subrahmanyan Chandrasekhar. Hydrodynamic and Hydromagnetic Stability. The International Series of Monographs on Physics. Clarendon Press, Oxford, 1961.

[3] Yuncherl Choi, Taeyoung Ha, Jongmin Han, and Doo Seok Lee. Bifurcation and final patterns of a modified Swift-Hohenberg equation. Discrete \& Continuous Dynamical Systems - B, 22(7):2543-2567, 2017.

[4] Pierre Colinet, Jean Claude Legros, and Manuel G. Velarde. Nonlinear Dynamics of Surface-Tension-Driven Instabilities. Wiley, first edition, May 2001.

[5] M. C. Cross and P. C. Hohenberg. Pattern formation outside of equilibrium. Reviews of Modern Physics, 65(3):851-1112, July 1993.

[6] Michael Cross and Henry Greenside. Pattern Formation and Dynamics in Nonequilibrium Systems. Cambridge University Press, 2009. 
[7] PC Dauby and G. Lebon. Bénard-Marangoni instability in rigid rectangular containers. Journal of Fluid Mechanics, 329(1):25-64, 1996.

[8] PC Dauby, G. Lebon, P. Colinet, and J.C. Legros. Hexagonal Marangoni convection in a rectangular box with slippery walls. The Quarterly Journal of Mechanics and Applied Mathematics, 46(4):683, 1993.

[9] H. A. Dijkstra. Pattern Selection in Surface Tension Driven Flows. In Hendrik C. Kuhlmann and Hans-Josef Rath, editors, Free Surface Flows, International Centre for Mechanical Sciences, pages 101-144, Vienna, 1998. Springer.

[10] Henk Dijkstra, Taylan Sengul, and Shouhong Wang. Dynamic transitions of surface tension driven convection. Physica D: Nonlinear Phenomena, 247(1):717, March 2013.

[11] Daozhi Han, Marco Hernandez, and Quan Wang. Dynamical transitions of a low-dimensional model for Rayleigh-Bénard convection under a vertical magnetic field. Chaos, Solitons \& Fractals, 114:370-380, September 2018.

[12] Daozhi Han, Marco Hernandez, and Quan Wang. Dynamic bifurcation and transition in the Rayleigh-Bénard convection with internal heating and varying gravity. Communications in Mathematical Sciences, 17(1):175-192, 2019.

[13] Marco Hernández and Kiah Wah Ong. Stochastic Swift-Hohenberg Equation with degenerate linear multiplicative noise. Journal of Mathematical Fluid Mechanics, 20(3):1353-1372, September 2018.

[14] Rebecca Hoyle. Pattern Formation: An Introduction to Methods. Cambridge University Press, Cambridge, 2006.

[15] Chanh Kieu, Quan Wang, and Dongming Yan. Dynamical transitions of the quasi-periodic plasma model. Nonlinear Dynamics, 96(1):323-338, April 2019.

[16] Yuri Kuznetsov. Elements of Applied Bifurcation Theory. Applied Mathematical Sciences. Springer-Verlag, New York, third edition, 2004.

[17] Marcello Lappa. Thermal Convection: Patterns, Evolution and Stability. John Wiley \& Sons, 2009.

[18] Limei Li, Marco Hernandez, and Kiah Wah Ong. Stochastic attractor bifurcation for the two-dimensional Swift-Hohenberg equation. Mathematical Methods in the Applied Sciences, 41(5):2105-2118, March 2018.

[19] Limei Li and Kiah Wah Ong. Dynamic Transitions of Generalized Burgers Equation. Journal of Mathematical Fluid Mechanics, 18(1):89-102, March 2016.

[20] Honghu Liu, Taylan Sengul, Shouhong Wang, and Pingwen Zhang. Dynamic transitions and pattern formations for a Cahn-Hilliard model with long-range repulsive interactions. Communications in Mathematical Sciences, 13(5):1289$1315,2015$.

[21] ChunHsien Lu, Yiqiu Mao, Quan Wang, and Dongming Yan. Hopf bifurcation and transition of three-dimensional wind-driven ocean circulation problem. Journal of Differential Equations, 267(4):2560-2593, August 2019.

[22] Tian Ma and Shouhong Wang. Bifurcation Theory and Applications, volume 53 of World Scientific Series on Nonlinear Science Series A. World Scientific, June 2005.

[23] Tian Ma and Shouhong Wang. Phase Transition Dynamics. Springer International Publishing, second edition, 2019.

[24] J. D. Murray. Mathematical Biology II: Spatial Models and Biomedical Applications. Interdisciplinary Applied Mathematics, Mathematical Biology. SpringerVerlag, New York, third edition, 2003.

[25] James D. Murray. Mathematical Biology: I. An Introduction. Interdisciplinary Applied Mathematics, Mathematical Biology. Springer-Verlag, New York, third edition, 2002. 
[26] Kiah Wah Ong. Dynamic transitions of generalized Kuramoto-Sivashinsky equation. Discrete and Continuous Dynamical Systems - Series B, 21(4):12251236, March 2016.

[27] J. R. A. Pearson. On convection cells induced by surface tension. Journal of Fluid Mechanics, 4(5):489-500, September 1958.

[28] Joseph Pedlosky. Geophysical Fluid Dynamics. Springer-Verlag, New York, second edition, 1987.

[29] Lambertus A. Peletier and Vivi Rottschäfer. Pattern selection of solutions of the Swift-Hohenberg equation. Physica D: Nonlinear Phenomena, 194(1):95126, July 2004.

[30] S. Rosenblat, S. H. Davis, and G. M. Homsy. Nonlinear Marangoni convection in bounded layers. Part 1. Circular cylindrical containers. Journal of Fluid Mechanics Digital Archive, 120(-1):91-122, 1982.

[31] S. Rosenblat, G. M. Homsy, and S. H. Davis. Nonlinear Marangoni convection in bounded layers. Part 2. Rectangular cylindrical containers. Journal of Fluid Mechanics, 120:123-138, July 1982.

[32] Taylan Sengul, Jie Shen, and Shouhong Wang. Pattern formations of 2D Rayleigh-Bénard convection with no-slip boundary conditions for the velocity at the critical length scales. Mathematical Methods in the Applied Sciences, 38(17):3792-3806, November 2015.

[33] Taylan Sengul and Shouhong Wang. Pattern formation in Rayleigh-Bénard convection. Communications in Mathematical Sciences, 11(1):315-343, 2013.

[34] Taylan Sengul and Shouhong Wang. Pattern formation and dynamic transition for magnetohydrodynamic convection. Communications on Pure and Applied Analysis, 13(6):2609-2639, July 2014.

[35] Martin B. Short, Andrea L. Bertozzi, and P. Jeffrey Brantingham. Nonlinear patterns in urban crime: Hotspots, bifurcations, and suppression. SIAM Journal on Applied Dynamical Systems, 9(2):462-483, 2010.

[36] J. Swift and P. C. Hohenberg. Hydrodynamic fluctuations at the convective instability. Physical Review A, 15(1):319-328, January 1977.

[37] Roger Temam. Infinite-Dimensional Dynamical Systems in Mechanics and Physics, volume 68 of Applied Mathematical Sciences. Springer-Verlag, New York, second edition, 1997.

[38] Huichao Wang, Quan Wang, and Ruikuan Liu. A time-dependent perturbation solution from a steady state for Marangoni problem. Applicable Analysis, 97(9):1526-1539, July 2018.

[39] Huichao Wang, Quan Wang, and Dongming Yan. On the stability and transition for the Navier-Stokes-alpha model. Mathematical Methods in the Applied Sciences, 43(5):2386-2402, 2020.

[40] Quan Wang and Dongming Yan. On the stability and transition of the CahnHilliard/Allen-Cahn system. Discrete $\&$ Continuous Dynamical Systems - B, 25(7):2607, 2020.

[41] Xige Yang and Dapeng Li. Analyzing Turing's Systems via Dynamic Bifurcation Theory. arXiv:1811.10031 [math], November 2018.

[42] Dongpei Zhang and Ruikuan Liu. Dynamical transition for S-K-T biological competing model with cross-diffusion. Mathematical Methods in the Applied Sciences, 41(12):4641-4658, August 2018.

Department of Mathematics, Marmara University, 34722 Istanbul, TURKEY

E-mail address: taylan.sengul@marmara.edu.tr 